\title{
Simulation-Based Clinical Skill Training to Promote Effective Clinical Learning with Simulation Evaluation Rubrics in Nursing Education
}

\author{
Alice M. L. Li
}

\begin{abstract}
Clinical simulation-based education can create a specific learning environment to ensure healthcare or nursing students to manage experiential learning by reinforcing their clinical skills through different levels of competency. This educational technology offers new avenues for supporting nursing students to experience high-fidelity simulation with lived experience on scenario-based clinical skill training that provides the better ways to support the nursing education. The use of simulation-based training needs to strengthen up students' clinical skills and practices in a more meaningful learning experience by using my newly inventive Simulation Evaluation Rubrics (SER). This paper illustrates this newly formed inventive SER by adopting Tanner's Clinical Judgment Model, combined with the constructed concept of 'Knowledge About knowledge' as a basis to specifically designed for the proposed conceptual model of inventive SER, which is used for capturing the effectiveness of clinical learning by monitoring the progress and benchmarking of performance outcomes, as well as providing invaluable informative feedback for further improvements on the students' competencies of nursing practice from the simulation-based clinical skill training.
\end{abstract}

Index Terms-Promote effectiveness of clinical learning, simulation-based clinical skill training, simulation evaluation rubrics.

\section{INTRODUCTION}

A long-standing development and focus of studies have been well documented with empirical evidence of the simulation-based clinical skill training that has been widely used to optimize the clinical learning activities in nursing education. Many research efforts have been verified the positive outcomes on the impacts of simulation-based tools for supporting various functions in clinical skill training for nursing education [1], [2].

Measurement of performance outcomes from the simulation-based clinical skill training is part of the vital role and process in educational evaluation, which requires the process of characterizing and appraising the aspects of learning as required and defined in accordance with the consistent application of learning expectations, which was generated from the Intended Learning Outcomes (ILOs) as part of quality assurance of the quality standards ensured under the paradigm of the Hong Kong Government's Qualifications Framework in Education.

The provision of feedback gathered from the evaluation of

Manuscript received August 20, 2014; revised October 23, 2014.

Alice M. L. Li is with HKU SPACE, Hong Kong (e-mail: alice.mla.li@hkuspace.hku.hk). the simulated clinical assessment practices is of significant for the students' experiential learning, as Oermann [2] has also echoed this aspect of importance and stated that "Excellence in nursing education requires evidence-based curricula, teaching approaches, and evaluation methods" [2]. This article create the concept of a newly inventive Simulation Evaluation Rubrics (SER) for evaluating the impact of simulation-based clinical skill training on various essentials and aspects such as communication, critical thinking, health assessment and nursing care interventions, etc.

Rubrics can in fact help students to see the connections between learning (what will be taught) and assessment criteria (what will be evaluated) by making use of the informative requirements as derived from the ILOs. In addition, the feedback generated from SER together with detailed quantitative and qualitative information and comments of identifying the students' outcomes of performance from simulated practices that can also communicated to students as part of debriefing process. For this reason, rubrics are generally designed to be simple, explicit, and easily understood for this intended purposes of evaluation, which can be used by different users, in terms of teachers, assessors, clinical instructors, as a way to maintain consistency and objectivity with valid informative comments for such intended purposes for professional usage in teaching, learning and educational assessments and evaluation of performance outcomes in clinical learning. This paper proposed a newly constructed conceptual model of inventive Simulation Evaluation Rubric by comprising the dimensions of Tanner's clinical judgment model with my constructed concept of 'Knowledge about Knowledge', so as to support this purposes, as well as for promoting and facilitating the effectiveness of clinical learning from the simulation-based skills training.

\section{Simulation-BASEd Clinical SkILl Training}

\section{A. Simulation for Practices - Connecting Education with Technology}

Safe practice is undoubtedly important in the training of nursing and health care professionals. Clinical reasoning and competency are the essential elements in safe practice. As Practice makes perfection of clinical skills, however, we cannot learn by trial and error in the actual real clinical settings. It is now possible and ethical to practice, with unlimited trails till the required clinical skills perfect, with the use of simulation-based clinical skill training, which is also supported by the World Health Organization [3]. 
Simulation-based training can provide pedagogical guidance to fulfill the required and intended learning outcomes through the cognitive instruction together with corrective feedback by using specifically designed Simulation Evaluation Rubric, which serves as a critical guide to inform and shape the nursing students' clinical learning.

Jeffries [4] postulated that "Simulations are defined as activities that mimic the reality of a clinical environment and are designed to demonstrate procedures, decision-making, and critical thinking through techniques such as role playing and the use of devices such as interactive videos or mannequins" [4], of which also echoed by Gaba [5] with further illustrations on affirming "simulation is a technique, not a technology, to replace or amplify real experiences with guided practices, often immersive in nature, that evoke or replicate substantial aspects of the real world in a fully interactive fashion". The IOM report on nursing work environments recommends simulation as a method to support nurses in ongoing acquisition of knowledge and skills [1]. The benefits of using simulation for the practices and experiential learning in clinical skills training are evidenced by the features as illustrated by Issenberg \& Scalese [6] that include the capabilities for:

- Repetitive practice

- Range of difficulty level progressively

- Multiple learning strategies

- Clinical variations

- Controlled environment or situation

- Individualized learning

- Defined outcomes and benchmarks

- Simulator realism with validity empowered

- Curricular integration

With all of these capabilities as mentioned and affirmed, simulation-based education has become more important technological and educational tools for nursing education. Subsequently, the crucial and central concern is of great consequence for nurse educator to formulate some guiding principles in effectively evaluating students' performance outcomes in clinical learning from their simulation-based clinical skill training and practices. One of the way to ensure the quality standard of assessment from the simulation-based clinical skill training is by using the 'Simulation Evaluation Rubric, in which we can communicate to our students ahead of time for what the required skills will be expected to know and do, together with a framework for the assessors to evaluate the students' work from this simulation-based practices, in turn, it also helps:

1) to eliminate some of the possible subjective views, components or expectations of such assessment;

2) to keep track of the processes in such assessment;

3) to allow this evaluation and feedback take place concurrently and/or then afterward as part of debriefing process from the simulation-based practices.

The linkages in the use of simulation evaluation rubric to evaluate clinical learning performance have been explored by many research studies throughout the last decade, with empirical results and findings generally suggesting and supporting better achievement and deeper or more effective learning by students who have rubrics to guide their work of the required expectations or intended learning outcomes and objectives [4], [7]-[9].

\section{B. Challenges in Designing Simulation Evaluation Instruments as Rubrics}

High quality assessment and evaluation of any clinical performance depends on accurate and reliable measurement of key performance indicators. Rubrics are tools that can assist clinical instructors/ assessors to measure students' ability to use and apply factual, conceptual, procedural and metacognitive knowledge in their simulated-clinical learning and skill training.

Normally, a rubric contains three essential features, namely 1) Evaluation criteria, 2) Quality definitions and 3) Scoring strategy as described in a review of rubric by Reddy and Andrade [10]. Under the feature of 'Evaluation criteria', which are the identified factors that an assessor considers when determining the quality of a student's work, whereas the 'Quality definitions' should provide a detailed explanation of what a student must do to demonstrate a required skills, in which is very similar feature of 'Focus on positive attainment' as suggested by Bargainnier in her talk on 'Fundamentals of Rubrics' [11]. The third feature of 'Scoring strategies' for rubrics involve the use of a scale for interpreting judgments of student's process of work. According to Arter and McTighe [12], rubrics can teach as well as evaluate, due to its potential to help students understand the intended learning outcomes and objectives for these targets, together with the required standards of quality for a specified knowledge and skill practice criteria.

The general principles and guidelines of rubric is an explicit set of criteria being formulated in accordance with the required standards for a specified knowledge and skill practice criterion for assessing a specific task. The rubric can be viewed as an ongoing part of formative assessment as both teachers and students should be involved into these processes. Rubrics usually divided into different levels of competency or achievement that typifies each required standards of criterion of required performance and that will be used as the basis to measure how well students perform by referencing to the pre-set requirements as criterion-referenced indicators for evaluation. Rubrics can also enable teachers to perform the comparison of students' performance outcomes in learning, and serve as evidential reference and criterion-based indicators for debriefing with feedback to students for their future improvements towards the expected quality standards of intended learning outcomes.

Kardong-Edgren, Adamson and Fitzgerald [13] postulated that the lack of reliable and valid instruments to evaluate simulation-based clinical learning outcomes is inhibiting the adoption and progress of simulation-based training in nursing education. They have signified the importance of educational evaluation, in terms of establishing acceptable validity and reliability standards, in which could make both teachers', clinical assessors' and students' expectation clear and are helpful for objectified on all areas of required clinical skills expected for students to work on [13]. A reliable and valid rubric should be able to:

- Reduce subjectivity and enhance objectivity and consistency for measurements across all students.

- Clarity for each criterion in specific terms. 
- Comply with the established ground rules for easy references in evaluating students' performance of specific-tasks as derived from the ILOs.

- Measure key aspects or critical items in relation to the required standard quality of learned knowledge-based components and skills practice.

The focuses of quality standards in designing, implementing, assessing and improving of all these areas as in the above-mentioned features and aspects have been well taken and embraced into my newly proposed conceptual model of 'Inventive Simulation Evaluation Rubrics' in this paper, in addition to the assurance of useful, feasible, intended and required criteria for the ultimate goals in promoting the effectiveness of simulated clinical learning as the expected standard quality derived from the ILOs of simulation pedagogies towards each specified task in using the simulated clinical practices for skill training.

\section{A NeWly Proposed InVentive SimUlation EVALUATION RUBRICS}

The development of any instrument for evaluating and assessing the clinical performance has to be based on the students' clinical judgment abilities and learning, in terms of the required component-based knowledge from the class lectures, together with integration of their learned skills from the simulation-based clinical training practices. In this regard, the measurement of the learning outcomes from this simulation evaluation rubric should be able to truly reflect students' 'holistic' learning as in the syllabuses of the curriculum. In addition, it is important that the SER can give the quality indications of outcome performance, as referred to in the required level and standard of the intended learning outcomes. This proposed conceptual model of inventive SER has adopted this necessity of reflections on the quality indications by accommodating such expected integrative 'holistic' learning into the built-in conceptual framework in this newly created conceptual model of an inventive simulation evaluation rubric.

In 2011, Davis and Kimble [14] conducted a research study, in which they have specifically acknowledged human patient simulation evaluation rubrics as an appropriate technological tool for meeting the educational needs of nurses in the $21^{\text {st }}$ century training, and perhaps more importantly, they have elucidated the importance and value of using simulation evaluation rubric to measure outcomes of human patient simulation as well as evaluates how these rubrics measure outcomes reflecting the required essentials for baccalaureate nursing education. As Aebersold and Tschannen [1] identified that in the past 20 years simulation has become more integrated into the education of nurses and physicians, it has not been as well integrated into the development of skills for practicing nurses. They further indicated that there is not currently one universally accepted framework or theory in use as a common guiding taxonomy and/or set of practices, despite the sciences of simulation education grows [1]. For this reason, this paper is also served in another proactive way to fill the gaps of this area, by putting forward this newly proposed conceptual model of an inventive SER as an alternative approach in the process to function as part of quality indicators in guiding the required standard criteria in simulated-based clinical learning, as derived from the ILOs, which also has to be reflected as central part of built-in concepts in this newly proposed model for rubric assessment that is based on Tanner's clinical judgment model and combined with my recent constructed concept of 'Knowledge about knowledge'.

\section{A. Tanner's Clinical Judgment Model}

Tanner [15] in 2006 proposed a 'Research-based Model of Clinical Judgment' that provides guidance to help students in the identified areas and consider learning experiences should be focused on the four aspects as follows:

1) "A perceptual grasp of the situation at hand, termed 'noticing'."

2) "Developing a sufficient understanding of the situation to respond, termed 'interpreting'."

3) "Deciding on a course of action deemed appropriate for the situation, which may include 'no immediate action', termed 'responding'."

4) "Attending to patients' responses to the nursing action while in the process of acting, termed 'reflecting'." [15].

She further illustrates that "Reviewing the outcomes of the action, focusing on the appropriateness of all of the preceding aspects (i.e. what was noticed, how it was interpreted, and how the nurse responded)." [15]. Tanner in 2009 also reinforced, yet again, clinical judgment requires deep background knowledge of central competencies for 1) noticing, 2) considering plausible interpretations, 3) collecting reasonable evidence and 4) choosing the best course of action [16].

Reflection on practice is often triggered by a breakdown in clinical judgment and is critical for the development of clinical knowledge and improvement in clinical reasoning. A model based on these general conclusions emphasizes the role of nurses' background, the context of the situation, and nurses' relationship with their patients as central to what nurses notice and how they interpret findings, respond, and reflect on their response in action. The developmental conceptual flow of Tanner's Clinical Judgment Model is shown in Fig. 1.

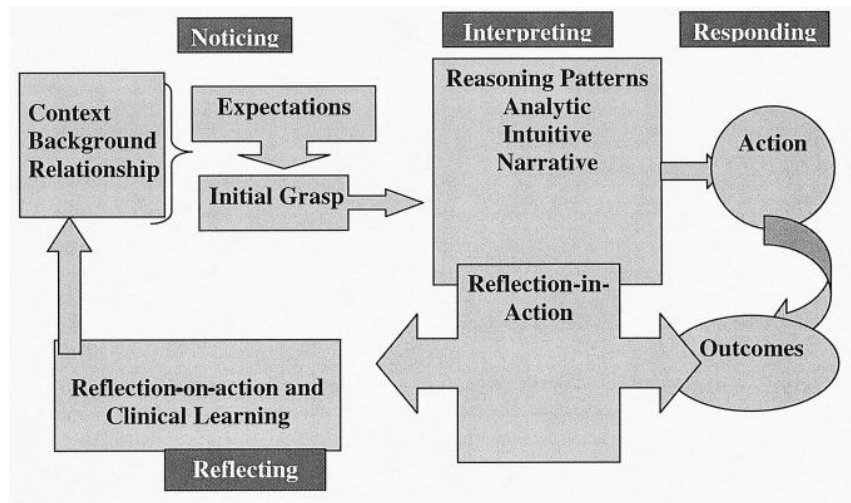

Fig. 1. Tanner's clinical judgment model [15]

Useful framework for instruction of the complexity of clinical judgment abilities and the many factors as arising from this Tanner's model is in the way to guide the expectation of instruction in performing clinical practices in 
nursing education. As Tanner described the different terms from the nursing literature such as 'clinical judgment, problem solving, decision making and critical thinking tend to be used interchangeably. And therefore, she has chosen the term of "clinical judgment" to mean an interpretation or conclusion about a patient's needs, concerns, or health problems, and/or the decision whether to take action or not [15].

In fact, good and relevant clinical judgements in nursing practice require various types of knowledge, that which could be further classified as in my proposed concept of "Knowledge About knowledge" that comprised of different aspects and component-based knowledge as elaborated in the following section.

\section{B. Concept of 'Knowledge about Knowledge'}

Knowledge is often defined as beliefs that are true and are justified. It is reasonable to think of a "true" belief as one that is in accord with the way in which objects, people processes and events exist and behave in the real world [17]. As Parker and Myrick [9] in 2010 postulated that "Transformative learning theory nurse educators with the tools to empower students to challenge their preconceived beliefs, assumptions, and values and socialize them appropriately to thrive in modern day clinical practice" (p. 326) [9]. Hunt [17] states that knowledge, itself, cannot be directly observed, it must be inferred from observing performance on a test, and therefore which has led to its measurement by methods, with rubrics in capturing the important dimensions of knowledge obtained, which allows evaluative measures of knowledge assessment and achievement [17], with which have been embraced and further developed in my proposed conceptual model of an inventive SER.

Toomey and Alligood [18] also echoed and mentioned that sound clinical judgment abilities improve with expanded knowledge and increased clarity for its purpose(s) that also proposed by the Nursing theorist Ernestine Wiedenbach. In my constructed concept of 'Knowledge About knowledge' that provides the educational implications on levels of satisfaction as required in simulated experiential nursing clinical practices, which was in place to help improve students' core competencies and to increase an effective clinical learning pedagogy for nursing education.

Use this concept of 'Knowledge About knowledge' to indicate the projected and planned criteria of different aspects of clinical learning that integrated with the specified knowledge-based components as quality indicators for different levels of competency in evaluating students' clinical judgment abilities. The required practice on specific-tasks as in 'Reflecting-on-action' skills performance for clinical practice as illustrated in Tanner's clinical judgment model that can further reflect the essentials of each specified task or criterion-based indicators, as according to the defined levels of competency, which was derived as the intended learning outcomes in the syllabuses under required aspects of the paradigm in the Hong Kong Government's Qualifications Framework for Education.

This article identifies different levels of knowledge-based components that are required as an important integral part of criteria for clinical learning and required dimension in measuring the performance outcomes as also reflecting from the Intended Learning Outcomes for clinical learning. And this proposed conceptual model created this significant dimension based on knowledge-based components in the concept of 'Knowledge about Knowledge' (KAK); and skills competencies as comprised with Tanner's Clinical Judgment Model, in which incorporated with the required clinical judgment abilities in performing practical skills as in Tanner's model and the component-based knowledge from the concept of KAK included:

1) "Declarative knowledge (knowledge-zero)

2) Cognitive knowledge (know what)

3) Procedure knowledge or Applied skill (know-how)

4) Reason knowledge or systematic understanding (know-why)

5) Condition-knowledge (know-when)

6) Relation-knowledge (know-with)

7) Affective Knowledge (ways to learn knowledge)" [19].

\section{A's Conceptual Model of Inventive Simulation Evaluation Rubric}

Each aspects of performance competency as expected and required from the component-based knowledge should be evaluated as according to the levels of achievement as described in Tanner's Model, namely as 'Noticing'; 'Interpreting'; 'Responding' and 'Reflecting'. This proposed conceptual model served and filled the gaps by synchronizing this significant dimension of requirements with the ILOs. This conceptual model is called A's conceptual model of Inventive Simulation Evaluation Rubric as shown in Fig. 2.

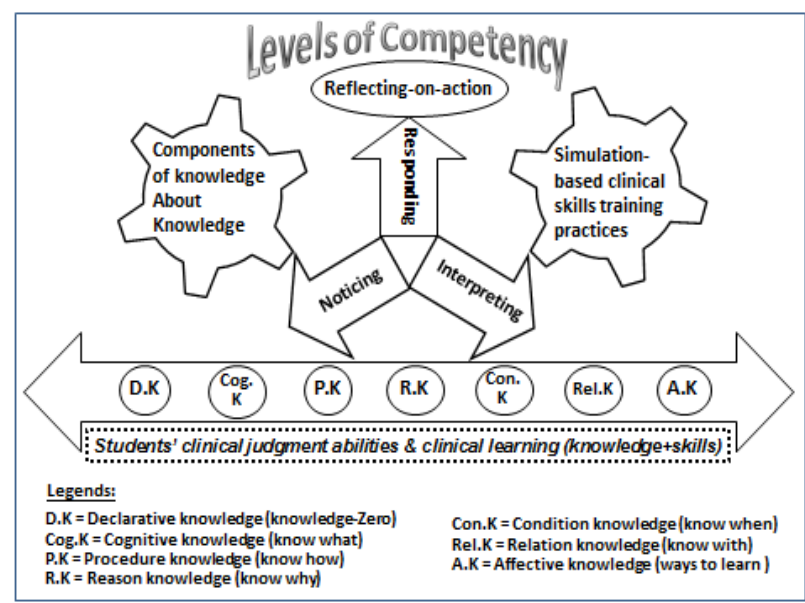

Fig. 2. A's conceptual model of inventive simulation evaluation rubric.

This conceptual framework connects the required aspects as derived from Tanner's model for clinical judgment abilities into the concept of 'Knowledge About Knowledge', which focused on different aspects of knowledge-based components and served as part of the integral tools for assessing different dimensions of knowledge components, so as to assess students' clinical judgment abilities and the required knowledge components for achieving the 'Reflecting-on-action' in the practical skills learning from the simulated-based clinical training. The ultimate goal of this A's Model is to advance the intended purposes of assessment agenda, with which it provides the platform and basis to summarise the grade of students' learning outcomes to further insure students can acquire skills and level of competency, as 
demanded and expected in the standard criteria of simulated clinical learning, as well as using the subsequent results evidentially to conduct debriefing and feedback to students for further improvements of performance outcomes. The resultant indications of students performance outcomes can assist to inform curricular modification or otherwise to empower teaching and learning in this area of simulated-based clinical skill training.

\section{Promote EfFective ClinicAl LeARning}

Simulation-based education has demonstrated benefits in clinical practice [1]. Students have a strong need for an assessment evaluation rubric to be used as part of the follow-on reflection from their simulation-based clinical training, which can provide more structured feedback that can be used as a further guide and indicators for the expected or intended learning outcomes. Luetke and Bembenek [20] pointed out that the results of assessment rubric also demonstrated for debriefing and verbal feedback that are essential components to students' learning experiences in a post simulation-based training session [20]. The benefit of the simulation evaluation rubric, in turn, provides additional areas of teaching outside of the standard simulation-based practices, including the ability to add evidence of a quality improvement from the invaluable informative feedback obtained from the assessment and this proposed conceptual framework of inventive SER also allows for the measurement of performance outcomes consistently and fairly with standard criterion as expected in accordance with the intended learning outcomes of clinical learning.

Luetke and Bembenek [20] also further recommended that nursing educators need to work to ensure simulation evaluation tools have standards equivalent to clinical evaluation tools if simulation is considered as part of the clinical experience training, which foster nursing students' transition into professional practice. Consistent use of well-designed rubrics significantly improves the facilitation of the effectiveness of clinical learning by providing both of students and instructors/ assessors with clarity and commonality of the intended purposes. Arter and McTighe [12] emphasized the importance for the rubric covers the features that can indicate quality performance for the relationship between performance criteria and rubrics, which in fact is the key to improve students' clinical learning.

The propriety of this proposed conceptual framework or model of Inventive Simulation Evaluation Rubrics is meant to be assisted for the endurances of these attributes and served as a quality indicators, as well as fulfilling the pre-set standard of evaluation guidelines that will be informative, timely and influential, with sufficient clarities, features and functions to justify the worthiness or merit of using this newly constructed conceptual model for the professional and educational evaluation of the simulation-based clinical skill training, which can also be used for the subsequent meaningful feedback to the students. Lasater [8] also found students desire feedback regarding their clinical performance in simulation-based clinical training, including potential simulated patient care outcomes, and also will listen to other students or their peers' experience and even their observation to other peers also promotes clinical reasoning skills in this respect.

In addition, this new model of simulation evaluation rubric embraced the concept of KAK that specifically allows the clinical instructors/assessors to assess students' think-on-action and think-in-action as illustrated in Tanner's Clinical Judgment Model [15] [16]. As Gantt [7] indicated the use of an evaluation tool is a way to increase meaning and deepen the learning experience for students, and the simulation grading rubrics allow assessing students' critical thinking and knowledge-based components of patient care scenarios in the context. The concept of 'Knowledge about Knowledge' with component-based aspects of knowledge is integrated for this particular purposes of assessing students' critical thinking with learned aspects of knowledge components, together with the clinical skill practices with think-on-action and think-in-action that can be reflected by using this newly created A's conceptual model of inventive SER. For this particular reason, the features of this A's model created the actual and potential capabilities with the functions included:

- Improve the teaching and learning processes in the simulated-based clinical skill training.

- Assess students' performance outcomes based on different required components of knowledge-based to achieve skills attainments in accordance with the required clinical judgments abilities as in the criterion-referenced indicators for measuring levels of competency in clinical learning.

- Make the comparisons of overall students' performance under the required intended learning outcomes.

- Provide useful and practical feedback on areas of strength and weaknesses.

- Benchmark against students' overall results with different areas of learning and each of its criterion can also be weighed to reflect the relative importance and priorities for each specific task.

- Provide a uniformly invaluable informational value for students' feedback and purposes for debriefing.

- Reflects the classroom learned knowledge and gaps between the clinical practices in the simulated-based clinical skill training.

- Contribute to the considerations of curriculum development and/or modifications.

- Promote an overall improvement and effectiveness of clinical learning.

\section{CONCLUSIONS}

In summary, this A's conceptual model of inventive simulation evaluation rubric operates as a tool to serve a vital function in generating relevant details with all the embracement of quality attributes in order to create and strengthen up the capabilities for evaluating the performance outcomes of simulated clinical practices and further promote the effectiveness of clinical learning from the use of simulation-based clinical skill training in nursing education.

This paper illustrates the significant role of professional and educational evaluation and rubric assessment by not just using for evaluation of students' simulated-clinical learning, but more importantly is to serve multiple functions in the 
educational aspects with the quality attributes from this conceptual model of simulation evaluation rubric, which project positive attainments with clear instructional criteria and assessment guidelines to insure the consistency of measurements by different clinical instructors or assessors across different time spectrum, as well as the provision of the subsequent feedback by making dependable judgments about students' performance of clinical learning as informed revision for further improvements in their clinical learning. Bargainnier [11] also supports that rubrics should be valid and reliable, with a valid rubric that measures key aspects central to quality of performance, while a reliable rubric yields consistent results for different users, which allows instructor to document the students' skills and progress of clinical learning fairly and consistently. In fact, simulation-based clinical skill training has been used for more than a decade, and these valuable avenues have been supported by many research studies as significant and meaningful technological and educational tools in healthcare and nursing education. As the proverb by Marcel Proust says that "The voyage of discovery lies not in finding new landscapes, but in having new eyes".

And this proposed A's conceptual model of inventive Simulation Evaluation Rubric is constructed to advance the way of assessment by integrating the concept of 'Knowledge about Knowledge' into Tanner's clinical judgment model as the conceptual basis and framework for the rubric assessment, in turn to improve teaching and learning outcome standards with benchmarked against the established quality requirements in assessing the performance of simulated-based clinical skills validly, reliably, fairly and consistently as the best practices of clinical assessment for the integrative 'holistic' clinical learning for the $21^{\text {st }}$ nursing educational standard guidelines of simulation evaluation rubric assessment, in which can be viewed as "having new eyes" for the "old" "landscapes" of the existed simulation-based clinical learning.

\section{REFERENCES}

[1] M. Aebersold and D. Tschannen, "Simulation in nursing practice: The impact on patient care," OJIN: The Online Journal of Issues in Nursing, vol. 18, no. 2, 2013.

[2] M. Oermann, "Evidence-based programs and teaching / evaluation methods: Needed to achieve excellence in nursing education," in $\mathrm{M}$. Adams and T. Valiga, eds., Achieving Excellence in Nursing Education, New York: National League for Nursing, 2009.

[3] World Health Organization, Nursing and Midwifery Human Resources for Health: Global Standards for the Initial Education of Professional Nurses and Midwives, Geneva: Author, 2009.

[4] P. Jeffries, "A Framework for designing, implementing, and evaluate simulations used as teaching strategies in nursing," Nursing Education Perspectives, pp. 96-103, 2005.

[5] D. Gaba, "The future vision of simulation in healthcare," Quality \& Safety in Healthcare, vol. 13, pp. i2-i10, 2007.
[6] S. B. Issenberg and R. J. Scalese, "Simulation in health care education," Perspectives in Biology \& Medicine, vol. 51, no. 1, pp. $31-46,2008$

[7] L. Gantt, "Using the Clark simulation evaluation rubric with associate degree and baccalaureate nursing students," Nursing Education Perspectives, vol. 31, no. 2, pp. 101-105, March / April 2010.

[8] K. Lasater, "Clinical judgment development: Using simulation to create an assessment rubric," Journal of Nursing Education, vol. 46, no. 11, pp. 496-503, 2007.

[9] B. Parker and F. Myrick, "Transformative learning as a context for human patient simulation," Journal of Nursing Education, vol. 49, no. 6, pp. 326-32, March 2010.

[10] Y. M. Reddy and H. Andrade, "A review of rubric use in higher education," Assessment and Evaluation in Higher Education, vol. 35, no. 4, pp. 435-448, July 2010.

[11] S. Bargainnier. (2003). Fundamentals of Rubrics. Faculty Development Series. [Online]. Available: http://www.webpages.uidaho.edu/ele/scholars/practices/Evaluating_P rojects/Resources/Using_Rubrics.pdf

[12] J. Arter and J. McTighe, Scoring Rubrics in the Classroom: Using Performance Criteria for Assessing and Improving Student Performance, Thousand Oaks, CA: Corwin Press, 2001.

[13] S. Kardong-Edgren, K. A. Adamson, and C. Fitzgerald, "A review of currently published evaluation instruments for human patient simulation," Clinical Simulation in Nursing, 2010, vol. 6, pp. e25-e35, 2010.

[14] A. H. Davis and L. P. Kimble, "Human patient simulation evaluation rubrics for nursing education: Measuring the essentials of baccalaureate education for professional nursing practice," Journal of Nursing Education, vol. 50, issue 11, pp. 605-611, November 2011.

[15] C. A. Tanner, "Thinking like a nurse: A research - based model of clinical judgment in nursing," Journal of Nursing Education, vol. 45, no. 6 , pp. 204-211, 2006.

[16] C. A. Tanner. (2009). The Future of Nursing Education: A Collaborative Perspective. School of Nursing, Oregon Health \& Science University. [Online]. Available: https://www.ncsbn.org/Tanner.ppt

[17] D. P. Hunt, "The concept of knowledge and how to measure it," Journal of Intellectual Capital, vol. 4, no. 1, pp. 100-113, 2003.

[18] A. Toomey and M. Alligood, Nursing Theorists and Their Work 6th ed., St. Louis, MO: Mosby, 2006.

[19] A. M. L. Li, "Reconceptualizing a creative and specific learning environment by using web-based automated e-quizzes as a guiding tools for accountable study behaviours," in Transforming Educational Practices with Technology, K. C. Li, T. L. Wong, S. Cheung, J. Lam, and K. K. Ng, (Eds)., 2014, Springer International Publishing AG.

[20] R. Luetke and B. D. Bembenek. (2012). Talk on "Simulation Evaluation: A Comparison of Two Simulation Evaluation Rubrics. [Online].

Available: http://www.qsen.org/docs/2012_conference/QSEN_2012_Luetke.pdf

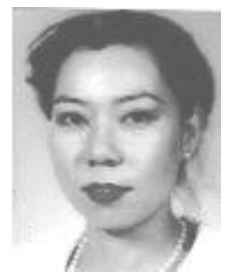

Alice Li was born in Hong Kong. She obtained her bachelor's degree in applied sciences and the master degree in public health from University of Sydney and University of New South Wales, Australia respectively. Her doctoral studies were in the field of health services management and policy-making for enhancing quality management of healthcare at the City University of Hong Kong.

She had been worked as an assistant professor at the Hong Kong Polytechnic University, and has been lived in the UK for more than a decade after married. She has settled back in HK currently and worked for HKU Space as a programme director. Her current and previous research interests include the areas in educational technologies for teaching and learning, healthcare-related issues and ecological public health matters. 